\title{
Cancer research: India meets the West
}

\author{
C Mandal ${ }^{1, *}$, S Roychoudhury ${ }^{1}$ and S Roy ${ }^{1}$ \\ Cell Death and Differentiation (2011) 18, 1675-1677; doi:10.1038/cdd.2011.85 published online 17 June 2011
}

30th Annual Convention of the Indian Association for Cancer (IACR) and The International Symposium on 'Signaling Network in Cancer'. CSIR-IICB, Kolkata, India, 6-9 February 2011

What can be more befitting than bringing a large number of cancer research experts from various parts of the globe to discuss recent advancements in complex cellular signaling network and cancer in a city that has more than 300-year-old rich history of culture and produced all nobel laureates from India. This meeting was organized by CSIR-IICB, which brought together several scientists from USA, UK, Canada, Germany, Japan, South Korea, Singapore, Italy and Australia, as well as a very large number of scientists from all over India to discuss emerging areas such as cancer cell signaling, signal networks, cell survival and novel or traditional therapeutic approaches to treat different cancers. The meeting was particularly enriched with the presence of large contingent of PhD students and research scholars from all over India, who presented their latest research findings in the area of cancer through more than 100 posters and 20 oral presentations. The meeting was as a part of the 30th Annual Convention of the Indian Association for Cancer Research (IACR) a premier body that promotes cancer research, education and awareness in India. IACR was founded in 1981 and is now a leading body of cancer research in India. It continues to attract people from diverse areas with a common interest in cancer.

It is indeed heartening to note that cancer research is being accorded top priority in India for developing drugs and technologies for the welfare of entire mankind. The conference began with the IACR presidential oration lecture by Rita Mulherkar. She discussed the genetics of cervical cancer and established that genes are expressed differentially in patients with no evidence of disease after treatment upon loco-regional metastasis or distant metastasis. Her study will provide clinicians with a set of molecular markers of prognosis and grading of the disease and prediction of response to radiation therapy. Mammen Chandy (Tata Medical Centre, Kolkata), in his keynote address, described some fascinating progress on the bone marrow-stem cell transplantation in different leukemic and thalassemia conditions applied in his center.

\section{Cell Cycle and Cell Death Regulation}

Mark Meuth (University of Sheffield, UK) explained the cell cycle regulation and its disruption in cancerous conditions. His major topic of interest was DNA damage response-mediated killing of tumor cells. He established a novel role of CHK1 in suppression of apoptosis and as a potential therapeutic target in cancer. Sagar Sengupta (NII, New Delhi) reported specific CHK1-dependent phosphorylation sites on BLM helicase following DNA damage.

WS El-Deiry (Penn state Hershey Cancer Institute, USA) illustrated targeted cell death in cancer therapy and discussed theories over $\mathrm{p} 53$ regulation in cancer. He also described novel strategies to develop anticancer agents, based on the p53related biochemical pathways. Gerry Melino (University Tor Vergata, Italy; Medical Research Council, UK), described the effect of the p53 family proteins, like p73 and p63; p73 null mice showed hippocampal dysegensis in the process of neuronal development. Here, p73 acts as a transcriptional activator of miR-34a. K Sakaguchi (Hokkaido University, Japan) explained abnormal nucleolar formation by overexpression of p53inducible protein phosphatase PPM1D, which modulated the nucleolar formation via nucleophosmin in breast cancer cells.

\section{Systems Biology Approach to Fight Cancer}

ET Liu (Genomic Institute of Singapore, Singapore), in his lecture entitled 'Genome-to-Systems Biology in Cancer Medicine', emphasized the need for systems biology approaches in cancer research and discussed the current ability to sequence cancer genomes in a cost-effective manner. This will allow identification of unique and private mutations for individual patient that will be helpful to develop personalized therapy against cancer. TJ Hudson (Ontario Institute for Cancer Research, Canada) illustrated the genomic variations in cancer in different human populations and elaborated aims of International Cancer Genome Consortium (ICGC). The aim is to generate comprehensive catalogs of genomic abnormalities in

\footnotetext{
${ }^{1}$ Infectious Diseases and Immunology Division, Council of Scientific and Industrial Research (CSIR)-Indian Institute of Chemical Biology (IICB), Kolkata, India ${ }^{*}$ Corresponding author: C Mandal, Infectious Diseases and Immunology Division, Council of Scientific and Industrial Research (CSIR)-Indian Institute of Chemical Biology (IICB), 4, Raja S.C. Mallick Road, Kolkata, West Bengal 700032, India. Tel: +91 332429 8861; E-mail: chitra_mandal@yahoo.com
} 
50 different cancer types and make the data available as rapidly as possible, with minimal limitations to scientists to accelerate research for management of cancer. PP Majumder (National Institute of Biomedical Genomic, Kalyani, India) also described ICGC and role of Indian initiatives to make it better and broader.

\section{Genetics, Epigenetics and Transcription}

K Somasundaram (IISc, Bangalore) explained epigenetics of glioma and astrocytoma development in relation to DNA methylation and microRNA regulation. On the epigenetic regulations, Subrata Sinha (NBRC, Haryana) described that heterochromatization and suppressing oncogene expression with consequent phenotypic changes resulting from the reactivation of the p53 and RB-mediated tumor suppressor pathways in cancer. M Bashyam (CDFD, Hyderabad) told that unique DNA lesions MLH1/MSH2 inactivation may account for a majority of non-polyposis colorectal cancer cases in India. SC Raghavan (IISc, Bangalore) and Manoj Mahimkar (ACTREC, Mumbai) described the chromosomal aberration and translocation in different cancerous condition and their potential role for the malignant propagation. Rajiv Kumar (German Cancer Research Center, Germany) demonstrated different genetic mutations of CDKN2A, PTEN, NRAS, EGFR, MITF, NOTCH2, CCND1, MDM2, CCNE1 and CDK4 genes in malignant melanoma. Similarly, CK Panda ( $\mathrm{CNCl}$, Kolkata) identified defect in several genes of EGFR, retinoblastoma and p53-associated pathways in oral cancer. Apart from the nucleus-associated genes, Bidyut Roy (ISI, Kolkata) illustrated the role of mitochondria and mitochondria-associated genes and significant association of SNP rs9905016 in the POLG2 gene with pre-cancerous stages of oral cancer. TK Kundu (JNCASR, Bangalore) illustrated histone hyperacetylation in $\mathrm{H} 3 \mathrm{~K} 14, \mathrm{~K} 9$ and $\mathrm{H} 2 \mathrm{AK} 5$ residues in oral cancer patients and claimed that histone acetylation inhibition could be a potential epigenetic target. Sanghamitra Banerjee (ISI, Kolkata) and Subrata Banerjee (SINP, Kolkata) shared their knowledge on the topic of genetics and genomics.

Transcription and transcriptomics was indeed a very hot field of interest. CK Sinha (University of Michigan, USA) elaborated his view on high-throughput transcriptome sequencing of cancers and ideas/possibilities to produce personalized medicine. Different transcription factors and their mode of action in cancers were explored by SK Manna (CDFD, Hyderabad) and Shantanu Chowdhury (CSIR-IGIB, New Delhi). They proved that Rel-A and NME2 transcriptionally controls cancer progression and metastases. S Chattopadhyay (NCCS, Pune) focused the role of SMAR1 as a transcriptional repressor in cancer, which modulated the transactivation potential of transcriptional co-activators NF- $\kappa \mathrm{B}$ and p53. SMAR1 facilitated DSB repair by assisting in anchorage of DNA repair factors at the site of repair and causing cell cycle arrest, allowing proper repair.

\section{Basic and Clinical Therapeutics}

Chitra Mandal (CSIR-IICB, Kolkata) illustrated the role of sialic acid in search of drug target. She demonstrated a significant correlation with newly induced 9-O-acetylated sialoglycoproteins/sialoglycolipids and sialate $O$-acetyl transferase enzyme with the high-risk acute lymphoblastic leukemia. Therefore, sialylation regulatory enzymes may be considered as potential target. S Raha (SINP, Kolkata) said that resveratrol targets key survival elements in cancer and causes apoptosis. TR Teni (ACTREC, Mumbai) illustrated radiation therapy in oral squamous cells and role of different apoptosis regulatory proteins (Mcl-1, Bcl-XI, survivin, p53 and p16). D Panda (IIT, Mumbai) showed that inhibition of microtubule dynamics blocks mitosis and retards cell division and migration and may consider as therapeutic target in cancer. Identification of disease marker through immunoproteomics was discussed by Surekha Zingde (ACTREC, Mumbai).

\section{Cancer Immunology and Signaling}

SV Chiplunkar (ACTREC, Mumbai) emphasized the role of notch and Toll-like receptors in regulating the effector functions of $\delta \gamma-T$ lymphocytes. Neeta Singh (AlIMS, New Delhi) established a synthetic peptide from HPV-16 to produce better immunoadjuvant activity in vaccination against cervical cancer. Anil Suri (NII, New Delhi) presented a firm relationship between SPAG9 protein expression and humoral immune response in majority of early-stage colorectal cancer patients, indicating that circulating anti-SPAG9 antibodies could be a novel serum biomarker for early diagnosis. B Mukherji (University of Connecticut, USA) talked about T-cell mediated cancer immune therapy. Arun Dharmarajan (The University of Western Australia, Australia) convincingly demonstrated the anti-angiogenic role of secreted frizzledrelated protein 4 and its possible therapeutic applications in the treatment of angiogenesis-dependent pathologies like cancer. MK Ghosh (CSIR-IICB, Kolkata) illustrated the cross talk of different signaling pathways like Wnt/ $\beta$-catenin and EGFR in glioma, breast and prostate cancer. Ellora Sen (NBRC, Haryana) described the role of TNF- $\alpha$ induced TLR4 in signaling events that sustained tumor promoting proinflammatory microenvironment of glioma. The newly-identified cancer stem cell was described by Tanya Das (Bose Institute, Kolkata) and she mainly demonstrated the signaling process by which a resistant cancer stem cell become prone to apoptosis. PS Dasgupta ( $\mathrm{CNCl}$, Kolkata) described that neurotransmitter dopamine act as an endogenous regulator of angiogenesis in cancer. In the absence of dopaminergic receptor, tumor growth was enhanced by angiogenesis suggesting dopamine as an anti-angiogenic factor. S Ashraf Imam (HMRI, USA) explained in vitro three-dimensional human breast cancer model, which consists of cancer, stromal cells and mimics tissue-like microenvironment. SG Rhee (EWHA Woman's University, Korea) discussed role of alcohol-induced oxidative injury of liver. SN Dalal (ACTREC, Mumbai) discussed about neoplastic progression and its relation with desmosome formation.

\section{Concluding Remarks}

The scope of cancer research is vast and expanding. This wonderful gathering provided a succinct update on current basic research in cancer biology and new therapeutic approaches in cancer treatment. Even though a number of mechanisms have been resolved, lots of questions still remain 
and more come up as novel thoughts emerged. As a result, the IACR platform truly emerged as the melting pot of novel ideas from East and West. The meeting ended with great optimism that the intense interactions among the participants would lead to more future collaborations.

Acknowledgements. This meeting was sponsored by CSIR, CSIR-IICB, Indian Medical Research Council, Department of Science and Technology, Govt. of
India. We thank all the national and local members of the organizing committee, treasurer, convenor and meeting attendees. We apologize to those researchers whose work may have been overlooked.

\section{Conflict of Interest}

The authors declare no conflict of interest. 